\title{
Isothermal fcc/hcp Transformation in Fe-Si-C-Alloy Thermally Treated at Lower Bainitic Transformation Temperature*
}

\author{
Kazuyuki Ogawa, Takahiro Sawaguchi and Setuo Kajiwara \\ National Institute for Materials Science, Tsukuba 305-0047, Japan
}

High resolution transmission electron microscopic observations have revealed that isothermal fcc $(\gamma)$ to hcp $(\varepsilon)$ transformation accompanies the bainitic transformation from $\gamma$ austenite to bcc $(\alpha)$ bainite in an Fe-2Si-1.4C (mass\%) alloy heated at lower bainitic transformation temperature of $570 \mathrm{~K}$. The $\varepsilon$ plates are formed at the ledges of $\alpha$ bainite $/ \gamma$ austenite interfaces. The unit volume of the $\varepsilon$ phase with respect to that of the $\gamma$ austenite increases by as much as $16 \%$ when the $\varepsilon$ plate has grown from $4 \mathrm{~nm}$ to $100 \mathrm{~nm}$ in thickness, probably due to enhancement of interstitial carbon. There is no visible stacking fault in the $\varepsilon$ phases. These experimental results are interpreted by the concept of bainitic transformation both with shear-type and diffusion-type nature. [doi:10.2320/matertrans.M2009262]

(Received July 31, 2009; Accepted September 4, 2009; Published November 25, 2009)

Keywords: isothermal transformation, hexagonal-close-packed structure, lower bainite, high resolution electron microscopy, atomic structure, interphase boundary, $S-N$ orientational relationship, $N$ orientational relationship, displacive transformation, diffusional transformation

\section{Introduction}

New steels taking advantage of bainite transformation have been developed recently, leading to renewed interest in the importance of bainite structure applications. ${ }^{1)}$ Bainite forms after isothermal process at between pearlite and martensite transformation temperatures. Structural characteristics separate upper bainite transformed in high temperature regime from lower bainite formed at low temperature. Bainite growth mechanism is still debated between "shear transformation theory" where bainite is considered to grow like martensite, ${ }^{2)}$ and "diffusion transformation theory", in which bainite grows like pro-eutectoid ferrite via movement of ledges at the interface in a process controlled by carbon diffusion. ${ }^{3)}$

To obtain direct evidence on bainite growth mechanisms using microscopy, we conducted high resolution transmission electron microscopy (HREM) of atomic arrangement in the interface between upper bainite or lower bainite and austenite in Fe-2Si-1.4C (mass\%) alloy. ${ }^{4)}$ In Fe-Si-C alloy systems, as bcc bainite grows, carbon atoms are forced to migrate to surrounding fcc austenite because of the silicon in the system, and as a result concentration of carbon in austenite increases. As a result, martensite transformation does not happen after quenching to room temperature. Thus, isothermal transformation in bainite temperature regime can be observed at room temperature, which is the reason we chose to observe this system. This system is favorable for interface observation because fine particles of carbides such as $\mathrm{Fe}_{3} \mathrm{C}$ do not precipitate, and plate-like structure formation can be observed. ${ }^{5)}$ Observations showed that lattice transformation region as seen in martensite/austenite interfaces does not exist in bainite/austenite interfaces, that misfit dislocations exist corresponding to the angle between the interface and $\{111\}$ terrace plane, and that high concentration of dislocations exist inside bainite, along with other features.

*This Paper was Originally Published in Japanese in J. Japan Inst. Metals 72 (2008) 1036-1043.
We propose that these observations can be consistently explained if bainite transformation is a combination of sheartype transformation and diffusion-type transformation. ${ }^{4)}$

In various observations of bainite transformation in $\mathrm{Fe}$ 2Si-1.4C (mass\%) alloy, we found an hcp structure phase in addition to bainite and austenite. Generation of the new hcp phase increases with processing time in lower bainite generation temperature range. We will call this phase $\varepsilon$ phase, although, as stated later, there is room for discussion whether this phase is the same as the $\varepsilon$ (hcp) phase that Jack discovered while tempering $\mathrm{Fe}-\mathrm{C}$ alloy martensite for the first time. In this report, we show results of direct observation with HREM of atomic arrangemnt at the interface between lower bainite, austenite and hcp phase forming in Fe-2Si$1.4 \mathrm{C}$ alloy (mass\%), and discuss formation mechanism of $\varepsilon$ phase relating to bainite formation mechanism reported previously. ${ }^{4)}$

\section{Experimental}

\subsection{Sample preparation}

Fe-2Si-1.4C (mass\%) alloys used were prepared by vacuum induction melting. After solution treatment for 10 minutes at $1420 \mathrm{~K}$, the samples were annealed for $570 \mathrm{~K}$ at 1 or $1.5 \mathrm{~h}$ to induce lower bainite transformation.

Samples for HREM observation were prepared using chemical polishing and electropolishing as follows. First, plate-like samples with $0.4 \mathrm{~mm}$ thickness were bainite transformed with above thermal processing conditions, then thickness reduced to less than $0.2 \mathrm{~mm}$ using chemical polishing with mixture of hydrofluoric acid, hydrogen peroxide, and water. Furthermore, the samples were cut into discs with $3 \mathrm{~mm}$ diameter using chemical polishing and electropolished with mixture of chromic acid, acetic acid and water while water cooling for use as HREM samples.

\subsection{HREM used for observation}

HREM with acceleration voltage $200 \mathrm{kV}$ (JEOL JEM2000EXII) was used for observations. Point resolution of 


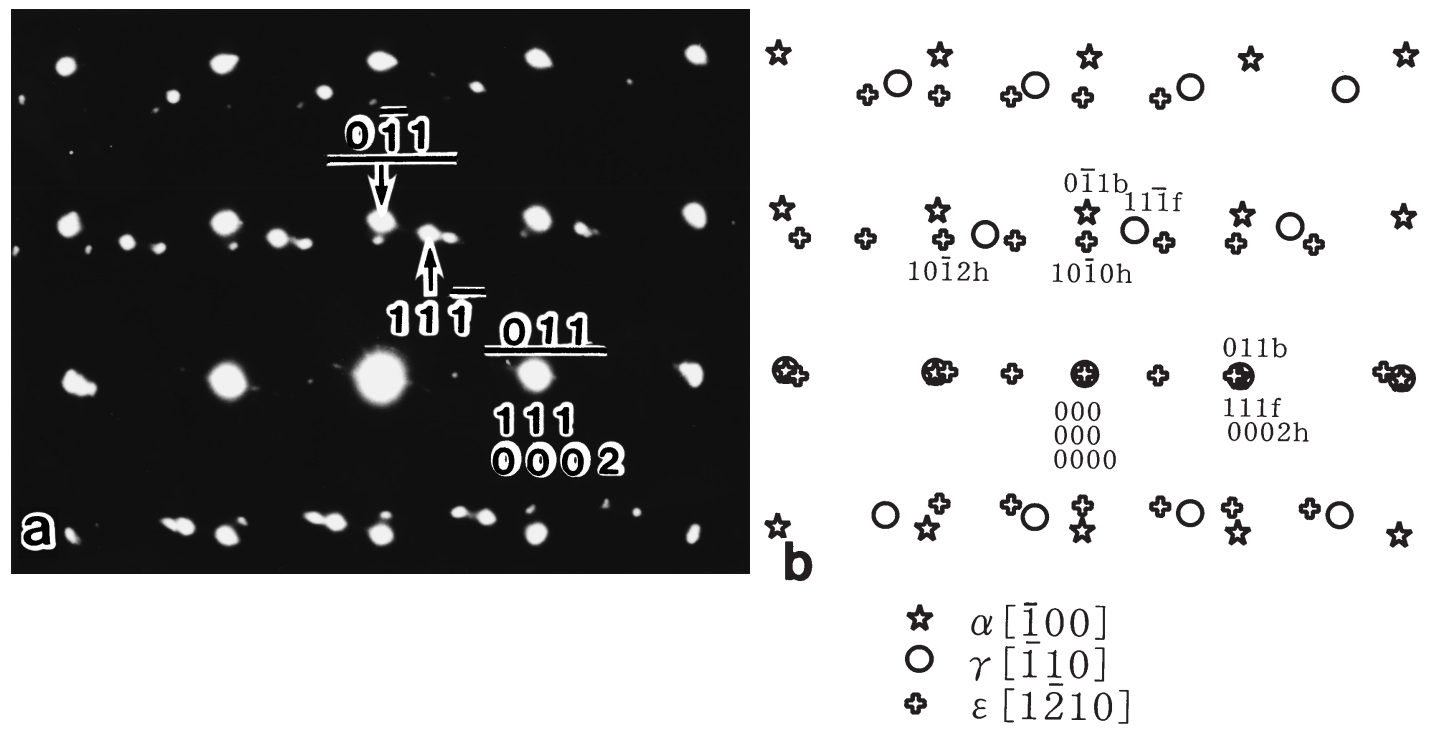

Fig. 1 (a) The diffraction spots taken from the area involving bcc $(\alpha)$ bainite and the hcp $(\varepsilon)$ phase and the fcc $(\gamma)$ austenite matrix. They are used for recording HREM images for alloys with the orientational relationship of $(111)_{\mathrm{f}} / /(011)_{\mathrm{b}} / /(0002)_{\mathrm{h}}$, [1 110$]_{\mathrm{f}} / /$ $[\overline{1} 00]_{\mathrm{b}} / /[11 \overline{2} 0]_{\mathrm{h}}$. (b) Key diagram for (a).

this microscope is $0.21 \mathrm{~nm}$, and the spherical aberration coefficient is $0.7 \mathrm{~mm}$. Observed HREM images were processed with Fast Fourier transformation (FFT). A limited number of spots were selected in the FFT pattern, and atomic configuration images were obtained by inverse FFT treatment of the selected spots.

\section{Results}

\section{1 $\varepsilon$ phase (hcp)}

$\mathrm{X}$-ray diffraction investigation of visible phases of $\mathrm{Fe}-2 \mathrm{Si}$ $1.4 \mathrm{C}$ (mass \%) alloy as a function of isothermal processing time between $1 \mathrm{~h}$ and $24 \mathrm{~h}$ at $300^{\circ} \mathrm{C}$ shows that the hcp phase starts forming about $1.5 \mathrm{~h}$ after onset of heating, and the quantity increases with processing time. At the same time, bainite (bcc) increases with processing time, and austenite decreases accordingly.

Figure 1(a) is an electron diffraction pattern showing formation of lower bainite (bcc) and $\varepsilon$ phase (hcp) in austenite, and Fig. 1(b) is the corresponding key diagram. Weak diffraction spots are from $\varepsilon$ phase (hcp). The diffraction image shows the orientational relationships between the phases as: $(111)_{\mathrm{f}} / /(011)_{\mathrm{b}} / /(0002)_{\mathrm{h}},[\overline{1} 10]_{\mathrm{f}} / /$ $[\overline{100}]_{\mathrm{b}} / /[11 \overline{2} 0]_{\mathrm{h}}$. However $(111)_{\mathrm{f}}$ and $(011)_{\mathrm{b}}$ are not exactly parallel but are $1.5^{\circ}$ apart.

Figure 2(a) shows schematically relations between lattices of $\gamma, \alpha$, and $\varepsilon$ phases. $\gamma$ and $\alpha$ phases exhibit the Almost Nishiyama orientational relation. $\gamma$ and $\varepsilon$ phases exhibit the Shoji-Nishiyama orientational relation. Figures 3(a) and (b) show the geometric relation when partial dislocations every 2 layers of the fcc (111) slip plane. Figure 3(a) shows the Burgers vector of a Shockley partial dislocation on the $(111)_{\mathrm{f}}$ plane, and (b) shows atoms in the fcc and hcp phases seen from the incident electron beam direction. Figure 3(c) shows an example of actually observed very thin hcp phase with six close packed planes. Figure 4 is a relatively low magnification image of the lattice taken with diffraction conditions of Fig. 1. Bainite $(\alpha)$ and $\varepsilon$ phases have formed in austenite, and

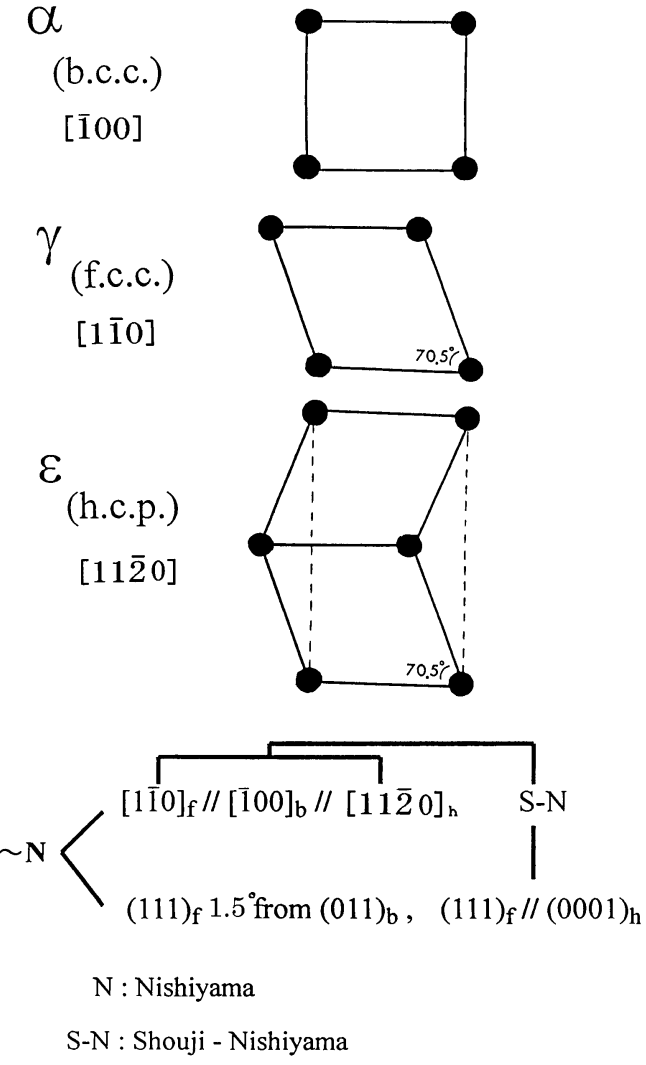

Fig. 2 Arrangement of atom rows projected along $[\overline{1} 10]_{\mathrm{f}},[\overline{1} 00]_{\mathrm{b}}$ and $[11 \overline{2} 0]_{\mathrm{h}}$, and the orientational relationship of fcc, bcc and hcp phases. The fcc and bcc phases exhibit the $\mathrm{N}$ orientational relationship, [110] $/ /$ $[100]_{b},(111)_{\mathrm{f}} / /(011)_{\mathrm{b}}$, and the fcc and hcp phases exhibit the S-N orientational relationship, $[\overline{1} 10]_{\mathrm{f}} / /[11 \overline{2} 0]_{\mathrm{h}},(111)_{\mathrm{f}} / /(0001)_{\mathrm{h}}$.

various kinds of interfaces can be observed, such as bainite forming next to austenite, $\varepsilon$ phase existing between two austenite regions, and the $\varepsilon$ phase also being in contact with bainite. In these interfaces, incident electron beam is not parallel except for very few cases, and Moire interference fringes are visible. 

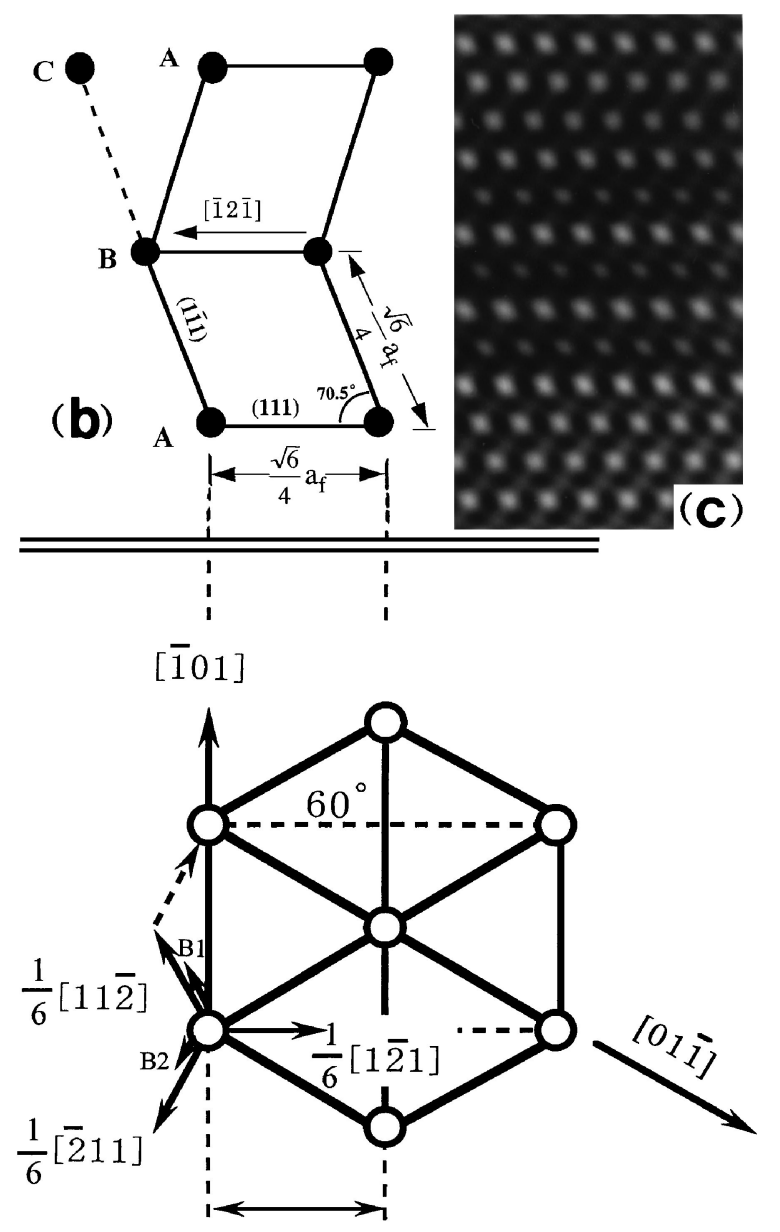

(a)

$$
\begin{aligned}
& (\sqrt{6} / 4) a_{f} \\
& \text { fcc }(111)
\end{aligned}
$$

Fig. 3 (a) Atomic arrangement of $(111)_{f}$ plane and a/6 $\langle 112\rangle$ Burgers vectors of Shockley partial dislocations on the plane. (b) Atomic arrangement of fcc and hcp phases seen from the direction of incident electron beam. (c) An experimentally observed hcp phase with the thickness of six atomic layers.

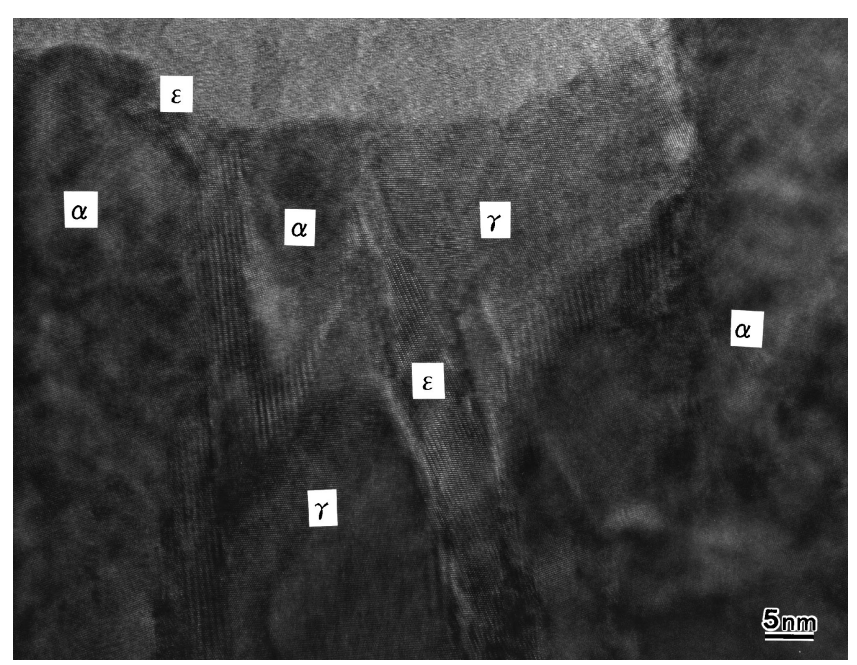

Fig. 4 Low-magnification HREM microstructure taken with the diffraction condition in Fig. 1, where the lower bainite and the $\varepsilon$ phase formed in the austenite.

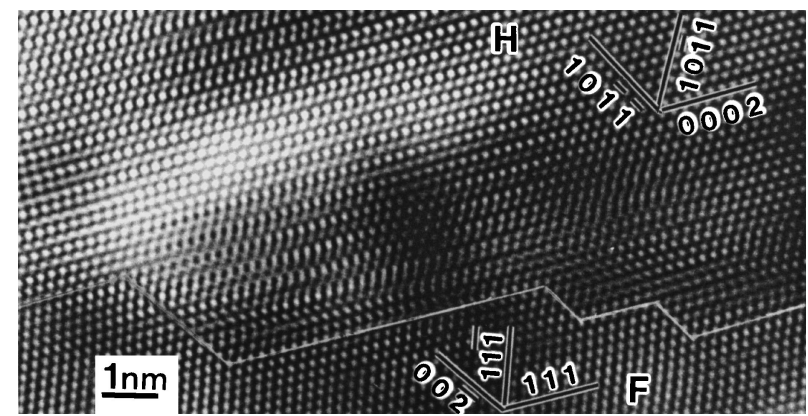

Fig. 5 FFT treated lattice image taken at the interface between the austenite and the $\varepsilon$ phase. The interface shows a ledge structure consisting of $(111)_{\mathrm{f}}$ and $(01 \overline{1})_{\mathrm{f}}$ planes.

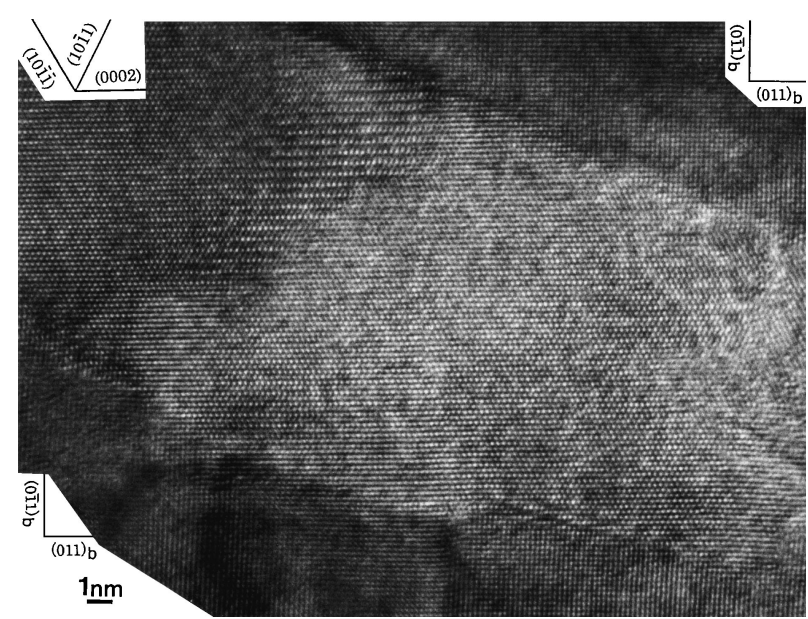

Fig. 6 A banded $\varepsilon$ phase formed in the $\alpha$ baininte, expanding in horizontal direction. The upper and lower parts of the figure is the bainite.

Figure 5 is a lattice image obtained by FFT treatment of high resolution electron microscope (HREM) images of the interface of $\varepsilon$ phase and austenite. The white line shows the interface of austenite and $\varepsilon$ phase. The $\varepsilon$ phase is seen above the white line and austenite is observed below. The interface of the two phases is made up of terrace structure $(111)_{\mathrm{f}} / /$ $(0002)_{\mathrm{h}}$ and ledge structure $(00 \overline{1})_{\mathrm{f}} / /(10 \overline{1} \overline{1})_{\mathrm{h}}$, forming a relatively large $(00 \overline{1})_{\mathrm{f}}$ ledge. Some lattice distortion is seen near the ledges of the interface; however the lattices of both phases are connected quite coherently.

Figure 6 is a relatively low magnification HREM image of $\varepsilon$ phase between bainite regions. A band of $\varepsilon$ phase is seen in the center of the image between bainite above and below. Contrast of lattice image of $\varepsilon$ phase is different in the right side and left side of the image. This arises from small differences in sample thickness and diffraction conditions.

Figure 7 is an enlarged image of Fig. 6 emphasizing the boundary between bainite above and $\varepsilon$ phase below. From the line showing the boundary in the image, we find that the interface is comprised of $(011)_{\mathrm{b}}\left(/ /(0001)_{\mathrm{h}}\right)$ terrace surfaces with $2 \sim 12$ atom rows, and $(01 \overline{1})_{\mathrm{b}}$ ledge surfaces with $1 \sim 5$ atom rows. As seen from the picture, the lattice of both phases are perfectly coherent, including at the ledges, and nowhere are the lattices incoherent. 


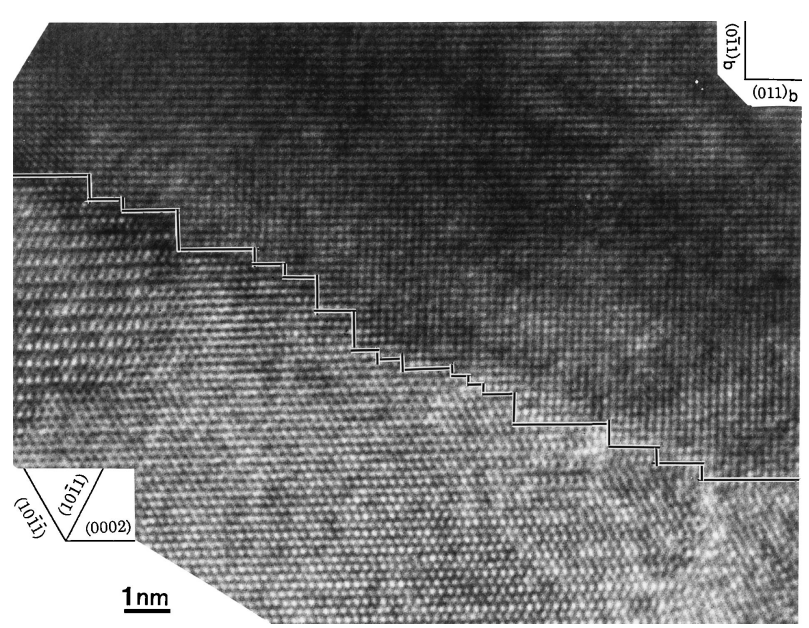

Fig. 7 The interphase boundary between the $\varepsilon$ phase and the bainite seen in the upper side of Fig. 6 . The ledge structure is delineated by the solid line in the figure.

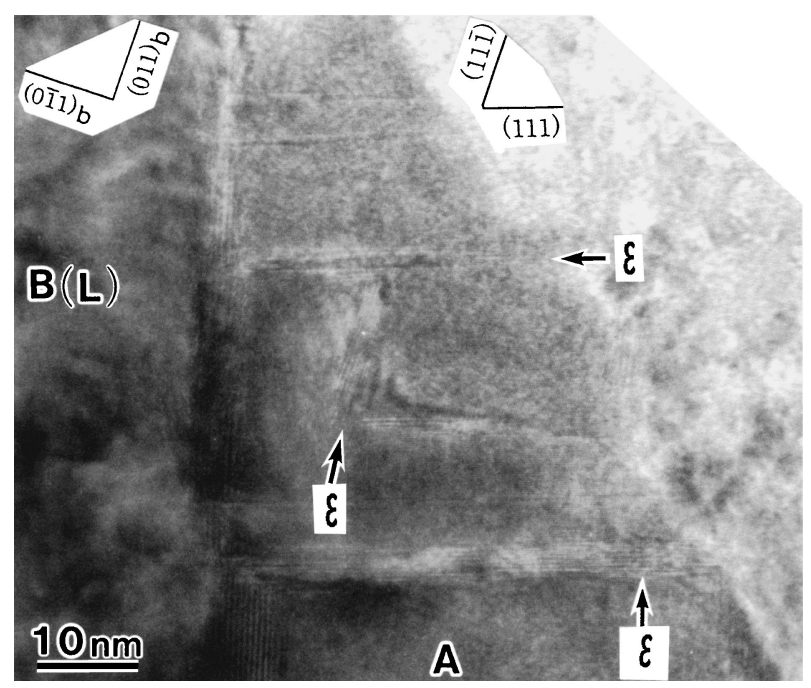

Fig. 8 The interphase boundary between the austenite/bainite interface. The banded $\varepsilon$ phase is formed in the austenite. Arrows indicate two kinds of $\varepsilon$ variants that are formed along the direction of the arrows.

Figure 8 is a low magnification HREM image where bainite $(\alpha), \varepsilon$ and austenite $(\gamma)$ phases were observed simultaneously. In this image, there is a direct interface between band-like $\varepsilon$ phase and austenite, and the thickness of the $\varepsilon$ plate is very small. Moire interference fringes were observed at the interface between austenite and bainite, showing the interface is not parallel to the incident electron beam. On the contrary, Moire interference fringes were not observed at the interface between austenite and $\varepsilon$ phase, proving that the interface is parallel to the incident electron beam. The band-like $\varepsilon$ phase forms between two different

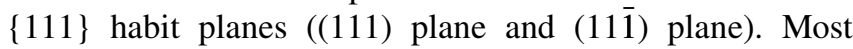
are (111) habit plane variants, observed in the horizontal direction in the figure, that is the direction of $\gamma / \alpha$ plane boundaries, and form at the $\gamma / \alpha$ phase boundary ledges. The orientational relationship between this $\varepsilon$ phase variant and $\alpha$ phase is different from that shown in Fig. 2, and the (0001) plane of $\varepsilon$ phase and $\{010\}$ plane of $\alpha$ plane is not parallel but is at an angle of $70.5^{\circ}$. At the center of Fig. 8, band-like

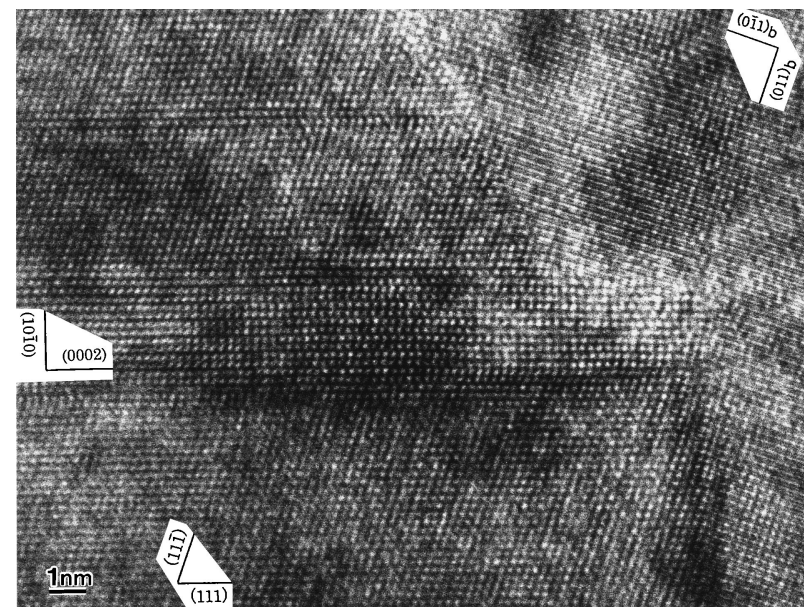

Fig. 9 The interface between the $\varepsilon$ phase and the austenite. The interface is parallel to the incident beam, and the coherence between both phases is clearly seen without Moire interference fringes.

$\varepsilon$ phase variants could be observed along (11) $\overline{1}$ ) plane of austenite. HREM observations of the $\varepsilon$ variant shows that the width of $\varepsilon$ phase is very narrow with only a few atom rows thickness, and grows by forming ledges, though it is not shown here.

Figure 9 is an HREM image of the phase boundary between $\varepsilon$ phase and austenite. Austenite and $\varepsilon$ phase are coherent at the interface, and Moire interference fringes are absent, meaning the interface is parallel to the incident electron beam. Bainite observed on the right is the same bainite variant as in the left side of Fig. 8, and the orientational relationship to the parent phase is $(11 \overline{1})_{\mathrm{f}} / /$ $(011)_{\mathrm{b}}$. The interface between austenite and bainite is tilted. Moire interference fringes could not be observed because the thickness of the area observed is very thin, resulting a relatively sharp image of the interface between two phases.

Figure $10(\mathrm{a})$ is a Fourier transformation pattern calculated for a region of $\varepsilon$ phase band about $4 \mathrm{~nm}$ wide and austenite next to the band of $\varepsilon$ phase. The arrows in Fig. 10(a) pointing upward show diffraction spots of the $\varepsilon$ phase. Figure 10(b) is a key diagram for the Fourier transformation pattern in Fig. 10(a) indicating Millar indices for diffraction spots of the $\varepsilon$ phase. Figure 11(a) is a Fourier transformation pattern similar to Fig. 10(a) prepared for $\varepsilon$ phase about $100 \mathrm{~nm}$ wide and neighboring parent austenite phase. In Fig. 11(b) we show again Fourier transformation patterns for $\varepsilon$ phase about $4 \mathrm{~nm}$ wide and neighboring parent phase to compare Fourier transformation patterns of $\varepsilon$ phase with different width. Comparison of the two figures show different positions of diffraction spots of the $\varepsilon$ phase relative to diffraction spots of the parent austenite phase, allowing us to investigate the effect of $\varepsilon$ phase thickness on hcp phase lattice. In Fig. 11(a), the hcp diffraction spots shown with arrows appear below the line linking $11 \overline{1} \mathrm{fcc}$ diffraction spots and the origin, and the position of the 0002 spots are closer to the origin than $111 \mathrm{fcc}$ spots. These observations show the hcp lattice has expanded. Similar trends are visible in Fig. 11(b), however the shift of spots are much smaller. In Fig. 11(a), the hep phase has expanded by $16 \%$ compared to corresponding fcc lattice as calculated from shifts in hcp spots. This implies that the unit 


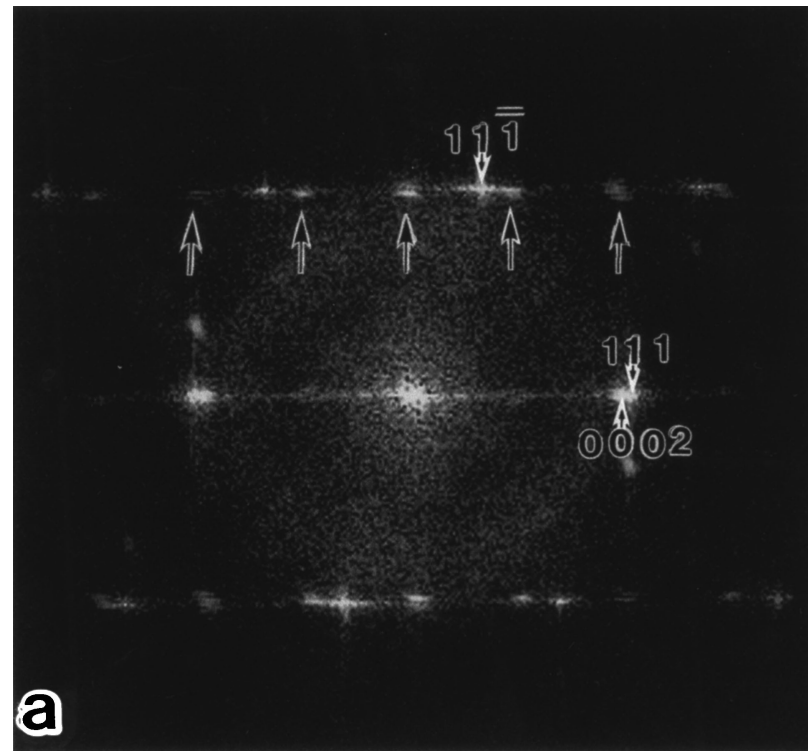

$[\overline{1} 10]_{\mathrm{f}} / /[1 \overline{2} 10]_{\mathrm{h}}$
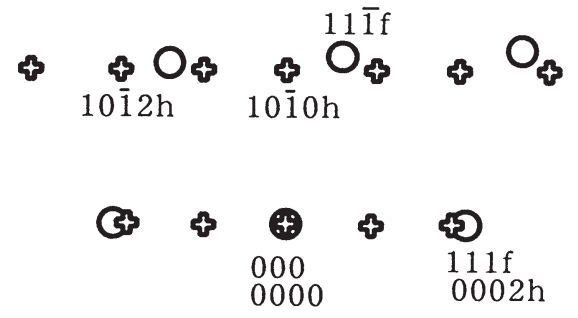

$0+\$ 0+0$ क क

b

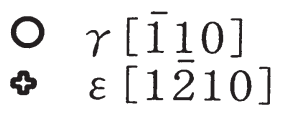

Fig. 10 (a) Diffraction pattern obtained by FFT calculation on an HREM image taken from the $\varepsilon$ phase with about 4 nm in width and the surrounding austenite. (b) Key diagram for (a). The diffraction spots of the $\varepsilon$ phase are indicated by upward arrows.

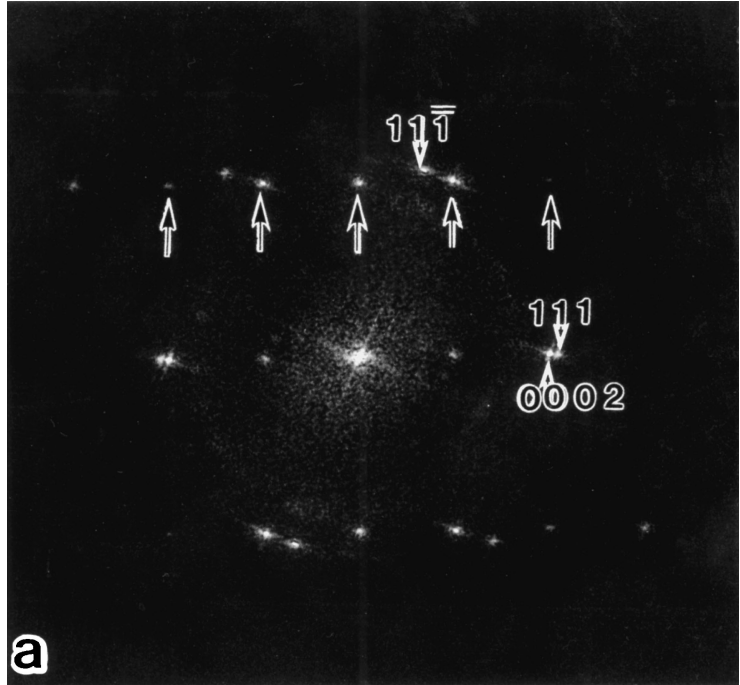

$[\overline{1} 10]_{\mathrm{f}} / /[1 \overline{2} 10]_{\mathrm{h}}$

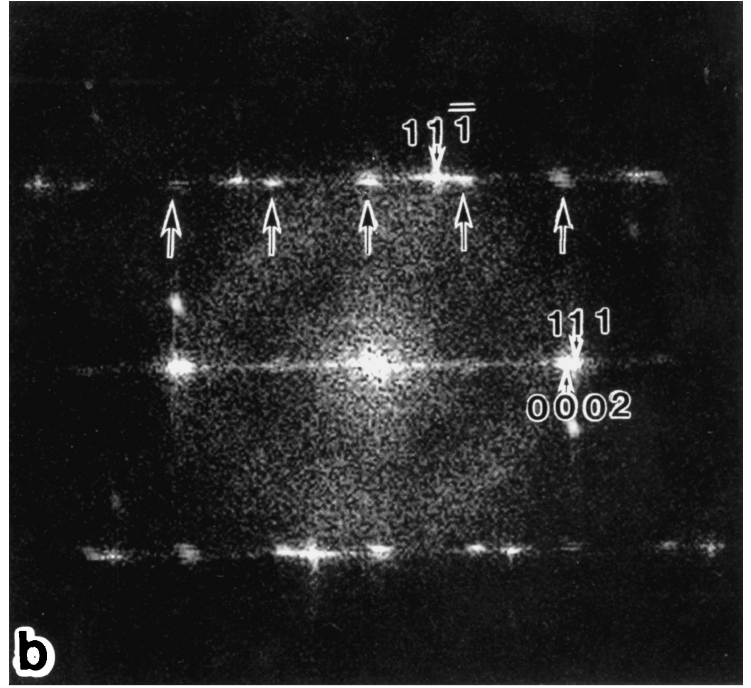

$[\overline{1} 10]_{\mathrm{f}} / /[1 \overline{2} 10]_{\mathrm{h}}$

Fig. 11 Diffraction pattern obtained by FFT calculation on an HREM image taken from the $\varepsilon$ phase with about (a) $100 \mathrm{~nm}$ and (b) $4 \mathrm{~nm}$, respectively, in width and the surrounding austenite.

cell of the lattice of $\varepsilon$ phase about $4 \mathrm{~nm}$ wide is $16 \%$ larger than $\varepsilon$ phase with width about $100 \mathrm{~nm}$. (Unit cell expansion was presumed to be zero in Fig. 11(b) because hcp diffraction spot shifts were small and difference in lattice size cannot be measured.) We believe that as hcp phase grows concentration of carbon increases gradually and lattice size increases.

Figure 12 is a blowup of very narrow $\varepsilon$ phase bands in austenite shown in upper side of Fig. 8. Note that the $\varepsilon$ plates bend at the places indicated by arrows, resulting in a shift of the (111) habit plane by a few atom rows. In particular, we can clearly observe a shift by two atom rows in the $\varepsilon$ phase above. The habit plane of upper $\varepsilon$ plate shifts downwards, while that of lower $\varepsilon$ phase shifts upwards. In this image, Moire interference fringes can be observed in the bainite/ austenite interface from the center to bottom, however in the bainite/austenite interface from the center to the top, Moire interference fringes are not visible, possibly because the thickness is very thin, allowing a clear view of the interface between two phases.

\section{Discussion}

The following is a summary of experimental results in this report.

(1) $\varepsilon$ phase (hcp) forms isothermally in temperature regime that causes lower bainite transformation. Extremely thin $\varepsilon$ phase in initial stages of formation has less carbon content compared to well-grown $\varepsilon$ phase. Such thin $\varepsilon$ 


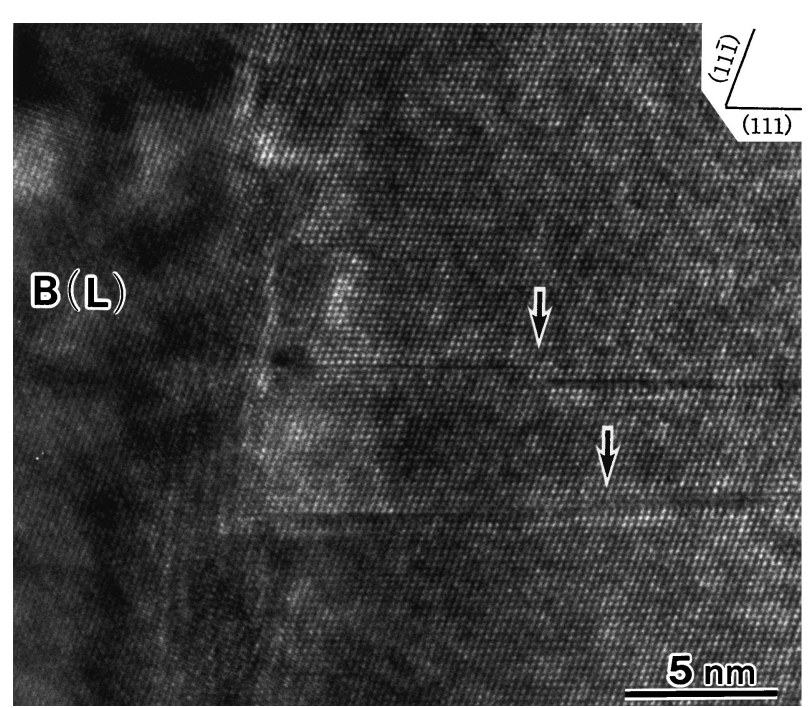

Fig. 12 Enlarged HREM image of Fig. 8 showing extremely thin $\varepsilon$ plates, which are bent at the places indicated by arrows and their habit plane shift by several atomic layers.

phase grows by forming ledges, with habit plane shifted by 1 to 2 layer shifts from $(111)_{\mathrm{f}}$ plane.

(2) Two types of orientational relationship of the $\varepsilon$ phase exist: One where (0001) plane is parallel to bainite (bcc) $\{110\}$ plane, and another where these two planes are at an angle of $70.5^{\circ}$. This is because $\varepsilon$ phase formed along two different $\{111\}$ planes in the austenite phase.

(3) In the latter case above, $\varepsilon$ phase forms from a large ledge at the $\gamma / \alpha$ phase boundary.

The authors have previously reported ${ }^{4)}$ that no lattice transition regime exists in the bainite/austenite interface observed in Fe-2Si-1.4C alloys as seen in martensite/ austenite interface, and that diffusional transformation-like features such as misfit dislocations corresponding to the angle between interface and $\{111\}$ terrace surface, and shear transformation-like characteristics such as high density dislocations inside bainite coexist. Furthermore, we have reported that bainite transformation mechanism combining shear transformation and diffusion transformation can consistently explain various structural observations claiming evidence of either diffusion or shear transformation, ${ }^{2,3,5-8)}$ by presuming alloy composition and processing temperature causes difference in whether shear transformation or diffusion transformation dominates. In particular, if upper bainite and lower bainite of the same alloy composition is compared, lower bainite forming at lower temperatures show more shear transformation-like characteristics.

The $\varepsilon$ phase discovered in our work may be considered as one type of bainite reaction because it forms from austenite by isotemperature process at relatively low temperature. From above experimental observation (1), the formation mechanism could be considered combined diffusion type and shear type bainite transformation. In other words, low carbon content in the thin $\varepsilon$ phase at start of formation is a result of shear type transformation, and diffusion type transformation must happen for carbon concentration to increase and the lattice to expand accompanying growth, and for shifting habit plane in order to grow.

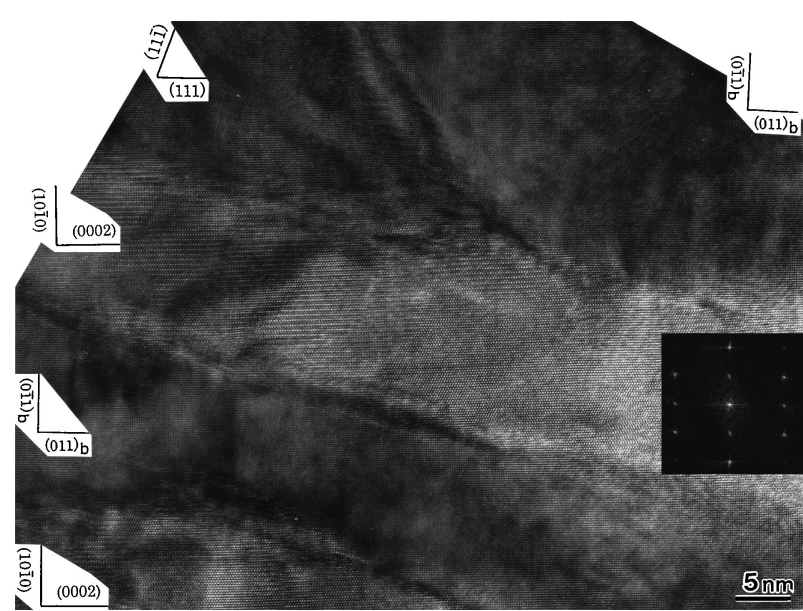

Fig. 13 HREM and the corresponding FFT images of the $\varepsilon$ phase, which show no stacking fault.

If the $\mathrm{fcc} \rightarrow$ hcp transformation occurs in a similar mechanism to diffusion/shear combined mechanism like bcc bainite formation, high density of dislocations can be expected inside hcp bainite ( $\varepsilon$ phase) generated by phase transformation as in bcc bainite ( $\alpha$ phase). Lattice defects in hcp structure are mainly stacking faults, however as seen in the lattice image of Fig. 13 very little stacking faults exist in the $\varepsilon$ phase observed in this research. This fact is supported by absence of streaks perpendicular to (0001) planes, which are specific to diffraction patterns from hcp phases including stacking faults, in FFT patterns shown in the insert. This result is a significant experimental finding.

As seen above, many observations imply fcc $\rightarrow$ hcp bainite transformation is dominated by diffusional processes. Observations suggesting effects of shear transformation is that $\varepsilon$ phase a few atom layers thick at start of formation has lower carbon concentration compared to $\varepsilon$ phase that are much thicker as described previously. Furthermore, one hcp phase variant inducing formation of other variants as seen in Fig. 8 may be an effect of shear mechanism.

Lattice defects from lattice invariant deformation are not observed although $\mathrm{fcc} \rightarrow$ hcp transformation starts like a shear transformation because $\{111\}$ planes of fcc phase acts as a habit plane and hcp (0001) planes forms parallel to the habit plane. As a result, the phase boundary automatically becomes a strain-free plane. In other words, lattice invariant deformation necessary to form a strain-free phase boundary in $\mathrm{fcc} \rightarrow$ bcc banite transformation is unnecessary in $\mathrm{fcc} \rightarrow \mathrm{hcp}$ bainite transformation. For fcc to hcp transformation, geometrically Shockley partial dislocations are necessary every two layers on $\{111\}$ planes, therefore such partial dislocations should exist in ledges of $\{111\}$ habit planes. Three orientations of Burgers vectors exist for Shockley partial dislocation $1 / 6 \mathrm{a}\langle 112\rangle$, and hcp phase with the same crystal orientation is formed regardless of the Burgers vector orientation. Furuhara et al. ${ }^{9)}$ observed fcc/hcp phase boundary of Al-Ag alloys using HREM and reported that three types of Shockley partial transitions are active. Strain accompanying $\mathrm{fcc} \rightarrow$ hcp phase transformation will be minimized when partial locations with these three Burgers vectors become active in turns. 
Next, we discuss formation of $\varepsilon$ phase and diffusion behavior of carbon atoms. As discussed in the previous section, initially the volume of $\varepsilon$ phase lattice is only slightly larger than the corresponding fcc lattice, however well grown $\varepsilon$ phase lattice is $16 \%$ larger than fcc lattice. This is caused by carbon atoms diffusing into interstices of the hcp lattice. These carbon are expelled into the austenite phase from bcc bainite as supported by the following evidence, therefore $\varepsilon$ phase is considered to form from bainite. The following evidence supports this assumption.

Two types of orientational relationships of $\varepsilon$ phase variants are observed in Fig. 8; $(111)_{\mathrm{f}} / /(011)_{\mathrm{b}} / /(0001)_{\mathrm{h}}$, and $(0001)_{\mathrm{h}}$ at an angle of $70.5^{\circ}$ from the former. In the latter variant, the orientational relationship $\{111\}_{\mathrm{f}} / /\{0001\}_{\mathrm{h}}$ holds, but $\{111\}_{\mathrm{f}} / /\{011\}_{\mathrm{b}}$ does not hold, clearly indicating direct formation from austenite (if formed from bcc bainite, angles between (0001) planes will be either $60^{\circ}$ or $90^{\circ}$ ). We described earlier that the former $\varepsilon$ phase variant forms on ledges at austenite/bcc banite interface. Close observation of how hcp and bcc lattices are connected show complete coherence as in Fig. 7. Therefore, the many ledges at the phase boundaries are expected to take an important role besides diffusion of carbon atoms. Bcc bainite expels carbon into the parent phase with growth, but on the contrary hcp phase is stabilized by carbon as seen in Fe-C alloys. As a consequence, sites where bcc bainite expels carbon may become preferential sites for nuclei formation of hcp phase. Formation of hcp phases at austenite/bcc bainite interfaces ledges suggests occurrence carbon diffusion through interface ledges.

We hereby explained the reason why, as seen in Fig. 13, carbon-rich hcp phase and carbon-deficient bcc bainite form alternatingly. The structure of hcp formed by this process and the structure of $\varepsilon$ phase discovered by Jack by tempering of carbon steel martensite ${ }^{10)}$ are considered to be different for the following reasons. First, the hcp phase found in Fe-2Si$1.4 \mathrm{C}$ alloy in this research has low carbon content in initial stages of formation but increases carbon concentration with growth, however the $\varepsilon$ phase Jack reported was precipitated $\varepsilon$ carbide from bct phase oversaturated with carbon. If $\varepsilon$ phase forms from bct martensite oversaturated with carbon, there is no need for carbon content to gradually increase, and it is more natural for the terminating carbon stoichiometry to be observed from the initial stages of formation. The second reason is how carbon atoms are located. In the latter $\varepsilon$ phase, the initial discoverer Jack determined the structure as hcp, however Hirotsu et al. ${ }^{11)}$ concluded, after detailed research using electron beam diffraction, that carbon atoms are ordered, the crystal system is orthorhombic, and the lattice has $\mathrm{Co}_{2} \mathrm{~N}$ ordering.

\section{Conclusion}

We conclude that hcp $(\varepsilon)$ phase found in Fe-2Si-1.4C after low temperature isothermal process had been formed by bainite reaction. This phase forms in austenite from austenite/bcc bainite interface ledges. Furthermore, the lattice expands and carbon content increases with growth of this phase. We presume nucleation is governed by shear type mechanism, but growth is controlled by diffusion type mechanism.

\section{Acknowledgement}

The authors would like to acknowledge Dr. Takehiko Kikuchi, National Institute for Materials Science for help in performing this study, Dr. Hisaki Okamoto at former Tottori University for providing with specimens and Prof. Yoshio Nakamura, Tokyo Institute of Technology, for his guidance and constant encouragement throughout the present work.

\section{REFERENCES}

1) T. Maki: Materia Japan 46 (2007) 321-326.

2) Y. Ohmori: Bull. Japan Inst. Metals 29 (1990) 542-551.

3) M. Enomoto and H. Tsubakino: Bull. Japan Inst. Metals 28 (1989) 732741.

4) K. Ogawa, T. Sawaguchi and S. Kajiwara: J. Japan Inst. Metals 72 (2008) 1028-1035.

5) H. Okamoto and M. Oka: Metall. Trans. A 17A (1986) 1113-1120.

6) M. Oka, H. Okamoto and K. Ishida: Metall. Trans. A 21A (1990) 845-851.

7) H. Okamoto: Osaka University Dissertation (1989).

8) T. Moritani, N. Miyajima, T. Furuhara and T. Maki: Scr. Mater. 47 (2002) 193-199.

9) T. Furuhara and T. Maki: Materia Japan 36 (1997) 483-490.

10) K. H. Jack: J. Iron Steel Inst. 169 (1951) 26-36.

11) S. Hirotsu and S. Nagakura: Acta Met. 20 (1972) 645-655. 